studies of thick films. By definition, a metastable substance such as $\mathrm{H}_{2} \mathrm{O}$ (as) will transform to a more stable phase on some time scale, though that may be so long as not to be of interest. For films estimated to be $c$. I ooo $\AA$ thick $\mathrm{H}_{2} \mathrm{O}$ (as) appears to be stable for $c .30 \mathrm{~min}$ at I6o K. It must be emphasized that the results I have described represent preliminary data-much more must be done to establish the validity of these observations. Accepting them to be correct, my interpretation is that a very thin film of $\mathrm{H}_{2} \mathrm{O}$ (as) contains fewer nucleation sites than does a thick film, hence the metastability range is extended, just as supercooling of water in a small capillary is more extensive than in a large capillary by virtue of exclusion of crystallization nuclei. It is conceivable that even thinner films of $\mathrm{H}_{2} \mathrm{O}$ (as) can persist for some minutes at still higher temperatures. Assuming the amorphous solid thermodynamic properties remain like those below $135 \mathrm{~K}$, part of the gap between the domains of metastability of supercooled water and $\mathrm{H}_{2} \mathrm{O}($ as $)$ is thereby closed. It remains an open question as to whether $\mathrm{H}_{2} \mathrm{O}$ (as) is, or is not, a metastable continuation of liquid water.

JoHARI: I am not certain whether the thermodynamic properties of $c$. I ooo $\AA$ thick layers (c. 360 water-molecules thick) would be the same as those of the bulk vitreous ice. I agree with you that more work on the properties of the material you describe is necessary.

J. Hallett: What is the evidence from "splat" (very rapid cooling by impact) studies that vitreous water can be obtained directly by cooling from temperatures about $-40^{\circ} \mathrm{C}$ ? It would appear that such experiments on supercooled drops at $-30^{\circ} \mathrm{C}$ might be worth taking.

Johari: I am aware of only one experiment by Professor Uhlmann which succeeded in obtaining vitreous water by splat cooling. His result has not been published in a paper but has appeared in the cited references in several papers on vitreous ice. Yes, I agree with you that the experiment you propose might be worth undertaking.

\title{
REFERENCE
}

Giguère, P. A., and Harvey, K. B. 1956. On the infrared absorption of water and heavy water in condensed states. Canadian Journal of Chemistry, Vol. 34, No. 6, p. 798-808.

\section{THE DIPOLAR CORRELATION FACTOR OF ICE VI}

\author{
By G. P. Johari and E. Whalley
}

(Division of Chemistry, National Research Council, Ottawa, Ontario K I A oR9, Canada)

Abstract. The dipole moment $\mu$ of an $\mathrm{H}_{2} \mathrm{O}$ molecule in the orientationally disordered ices is greater than the moment of an isolated molecule, I. $84 \mathrm{D}$, due to two factors: (i) the mutual polarization of molecules, and (ii) the short-range average correlation of the dipole vectors, if the molecules are able to reorient. The magnitude of the average enhancement is given by Kirkwood's equation,

$$
\left(\epsilon_{0}-\epsilon_{\infty}\right)=2 \pi \mathcal{N} g \mu^{2} / k T,
$$

where $\epsilon_{0}$ and $\epsilon_{\infty}$ are respectively the limiting low- and high-frequency relative permittivity of orientational polarization, $\mathcal{N}$ is the number density of dipoles, $k$ is the Boltzmann constant and $T$ is the temperature. The dipolar correlation factor $g$ is defined by,

$$
g=\lim _{\substack{r \rightarrow \infty \\ R / r \rightarrow \infty}}\left\langle\sum \mu_{i} \cdot \mu_{j}\right\rangle / \mu^{2},
$$


where $\mu_{i}$ and $\mu_{j}$ are the dipole moments of molecules $i$ and $j$ in a spherical region of radius $r$ immersed in a larger spherical region of radius $R$ and the angular brackets indicate average over all molecules $i$ and all their orientations. In view of the defects, vacancies, imperfections, etc., in ice, it is not certain if the limits in Equation (2) have any experimental significance. However, an approximate value of $g$ can be calculated by taking molecules up to the second co-ordination shell.

The theoretical value of $g$ for polycrystalline ice VI which is fully disordered (within the restrictions of ice rules) has been calculated by taking molecules up to the second nearest neighbours, in both the dendritic and non-dendritic models. The calculations were done for two kinds of molecules, I and II, having multiplicities 2 and 8 respectively, in the tetragonal unit cell in space group $P_{42} / n m c$, and, through the use of symmetry, keeping to a minimum the number of scalar products.

In both the dendritic and non-dendritic models, $g^{\mathrm{I}}$ is $10 \%$, or more, greater than $\mathrm{gII}^{\mathrm{II}}$ and the appropriately weighted values of $g$ are 2.342 in the former and 2.065 in the latter model. The effect of ring closure is, therefore, to reduce the value of $g$ by $13 \%$. The limiting high temperature experimental value of $g$ from Equation (I), using Onsager's theory for the enhancement of the dipole moment by its own reaction field, is 2.42 (Johari and Whalley, 1976). By analogy with the $10 \%$ discrepancy between the theoretical and experimental values of $g$ of ice I, a better estimate of the experimental $g$ of ice VI (using a reaction field other than that given by the Onsager theory) would be about $10 \%$ less than this value. Thus the suggested experimental $g$ of ice VI (2.18) is close to the theoretical values obtained from either of the two models and there is little contribution to $g$ from neighbours beyond the second co-ordination shell.

\section{REFERENCE}

Johari, G. P., and Whalley, E. 1976. Dielectric properties of ice VI at low temperatures. Fournal of Chemical Physics, Vol. 64, No. in, p. $44^{8} 4^{-89}$.

\section{DISCUSSION}

W. B. KamB: In doing the calculations, did you place the protons directly on the O-O centrelines, or somewhat off these lines as they really must be because the $\mathrm{O}-\mathrm{O}-\mathrm{O}$ bond angles are far from the $\mathrm{H}-\mathrm{O}-\mathrm{H}$ angle of the water molecule?

G. P. Johari: We placed the protons directly on the $\mathrm{O}-\mathrm{O}$ centre-lines only for the sake of convenience in our diagrams of projection of the unit cell of ice VI. The protons must of course be off these lines. In our calculations it does not matter where you place them in relation to the $\mathrm{O}-\mathrm{O}$ centre-line, as long as the dipole moment of a given water molecule, which is directed along the bisector of $\mathrm{H}-\mathrm{O}-\mathrm{H}$ angle, is parallel to the bisector of the $\mathrm{O}-\mathrm{O}-\mathrm{O}$ angle.

J. F. NAGLE: I would like to follow up your brief comments about the anisotropy. Is there enough geometrical anisotropy in ice VI to show up in a calculation of $g$ that goes only as far as second neighbours?

Johari: Yes, it seems there is. There are two four-membered rings for molecules of type I (which lie on the four-fold axis) and three four-membered rings for molecules of type II (which lie on the symmetry point $\mathrm{m}$ ). These rings extend to second neighbours. 